\title{
Radial-Fluctuation-Induced Stabilization of the Ordered State in Two-Dimensional Classical Clusters
}

\author{
I. V. Schweigert,* V. A. Schweigert,* and F. M. Peeters ${ }^{\dagger}$ \\ Departement Natuurkunde, Universiteit Antwerpen (UIA), B-2610 Antwerpen, Belgium
}

(Received 3 December 1999)

\begin{abstract}
Melting of two-dimensional (2D) clusters of classical particles is studied using Brownian dynamics and Langevin molecular dynamics simulations. The particles are confined either by a circular hard wall or by a parabolic external potential and interact through a dipole or a screened Coulomb potential. We found that, with decreasing strength of the interparticle interaction, clusters with a short-range interparticle interaction and confined by a hard wall exhibit a reentrant behavior in its orientational order.
\end{abstract}

PACS numbers: $64.60 . \mathrm{Cn}, 83.20 . \mathrm{Hn}$

The structural and dynamical properties of small classical two-dimensional (2D) clusters have been the subject of recent experimental studies [1-3] and Monte Carlo and molecular dynamics simulations [4-9]. It was found earlier that the particles are arranged in shells and that melting of finite clusters is a two step process. With increasing temperature, intershell motion develops and the system loses angular order. Consecutively, radial diffusion switches on and destroys the shell structure of the cluster. The spectrum of such clusters was obtained in Refs. [6,7] for different number of particles. The derived minimal frequencies and the corresponding energy barriers showed that "non-close-packed" clusters are unstable against intershell rotation. High symmetry clusters (i.e., the so-called magic number clusters) have energy barriers for intershell motion which are several orders of magnitude larger than those for non-close-packed clusters.

Recently, Bubeck et al. [3] observed reentrant melting in two-dimensional (2D) colloidal clusters. The clusters consist of paramagnetic colloidal spheres which were confined in a circular hard wall vessel. The external magnetic field induces a magnetic moment $\vec{M}$ in the particles and they interact through a dipole potential $V\left(\vec{r}_{i}, \vec{r}_{j}\right)=\mu_{0} M^{2} / 4 \pi r_{i j}^{3}$, where $\mu_{0}$ is the magnetic permittivity, and $r_{i j}$ is the interparticle distance. The coupling parameter, which is the interparticle interaction energy measured in units of the particle kinetic energy $\Gamma=V / k_{B} T$, characterizes the order of the system. It decreases by lowering the external magnetic field. In Ref. [3] it was found that, with decreasing $\Gamma$, first intershell rotation appears which destroys the angular order of the cluster. Further decreasing the parameter $\Gamma$, the system unexpectantly regained angular order within a narrow range of $\Gamma$ and then melts when $\Gamma$ is further decreased. It was suggested that the observed reentrant melting behavior is due to the increasing role of the radial particle fluctuations which is similar to an earlier investigation of laser induced melting of 2D colloidal crystals $[10,11]$.

Earlier theoretical work [5] on parabolic confined clusters did not find such a reentrant behavior which suggests that the shape of the confinement potential may be very important. Another difference is that in the experimental system the particle motion is strongly damped because the colloidal particles move in water. In the present paper we investigate the mechanism for the reentrant behavior and address the specific role played by the type of confinement (i.e., hard wall versus parabolic) and of the functional form of the interparticle interaction (short range versus long range) on the melting of the clusters.

In our model the particles are confined by a circular hard wall potential $\left(V_{p}=0\right.$ for $r \leq R$ and $V_{p}=\infty$ at $r>R$ ) or by a parabolic potential $V_{p}=\alpha r^{2}$. The particles interact through a dipole potential $V\left(\vec{r}_{i}, \vec{r}_{j}\right)=q^{2} /\left|\vec{r}_{i}-\vec{r}_{j}\right|^{3}$, where $q^{2}=\mu_{0} M^{2} / 4 \pi$, or through a screened Coulomb potential $\quad V\left(\vec{r}_{i}, \vec{r}_{j}\right)=\left(q^{2} /\left|\vec{r}_{i}-\vec{r}_{j}\right|\right) \exp \left(-\kappa\left|\vec{r}_{i}-\vec{r}_{j}\right|\right)$, where $q$ is the "particle charge," $\vec{r}_{i}$ is the coordinate of the $i$ th particle, and $1 / \kappa$ is the screening length where $\kappa=$ 0 for a Coulomb cluster, and we took $\kappa=2 / a_{0}$ for the screened Coulomb cluster where $a_{0}$ is the mean interparticle distance. For a given type of interparticle interaction and external confinement, only two parameters characterize the order of the system: the number of particles $N$ and the coupling parameter $\Gamma$. We define the characteristic energy of interparticle interaction for dipole clusters as $E_{0}=$ $q^{2} / a_{0}^{3}$ and $E_{0}=q^{2} / a_{0}$ for screened Coulomb clusters, where $a_{0}=2 R / N^{1 / 2}$ for the hard wall and $a_{0}=$ $q^{2 / 5} \alpha^{-1 / 5}$ for parabolic confinement. In the present calculation, we define the coupling parameter as $\Gamma=$ $q^{2} / a_{0}^{3} k_{B} T$ for dipole clusters and $\Gamma=\left(q^{2}\right)$ $\left.a_{0} k_{B} T\right) \exp \left(-\kappa a_{0}\right)$ for screened Coulomb clusters. In [3] a different dimensionless parameter $\Gamma$ was introduced, where $V$ was taken to be the sum over all pairs of particles. Our coupling parameter $\Gamma$ is a factor 2.2447 smaller than the one of [3] for $N=29$.

The ratio of the particle velocity relaxation time versus the particle position relaxation time is very small due to the viscosity of water and therefore the motion of the particles is diffusive. In our simulations we will neglect hydrodynamic interactions. Following [12] we rewrite the stochastic Langevin equations of motion for the position of the particles as those for Brownian particles: 


$$
\frac{d \vec{r}_{i}}{d t}=\frac{D_{0}}{k_{B} T}\left(\sum_{j=1}^{N} \frac{d V\left(\vec{r}_{i}, \vec{r}_{j}\right)}{d \vec{r}}+\frac{d V_{p}\left(\vec{r}_{i}\right)}{d \vec{r}}\right)+\frac{\vec{F}_{L}}{m},
$$

where $D_{0}$ is the self-diffusion coefficient, $m$ is the particle mass, and $\vec{F}_{L}$ is the randomly fluctuating force acting on the particles due to the surrounding media. In the numerical solution of Eq. (1) we took a time step $\Delta t \leq 10^{-4} /\left(n D_{0}\right)$, where $n=N /\left(\pi R^{2}\right)$ is the particle density and $\Delta t$ was varied within a range $(0.02-0.05) \mathrm{s}$. The radius of the circular vessel $R=36 \mu \mathrm{m}$ and the self-diffusion coefficient $D_{0}=0.35 \mu \mathrm{m}^{2} / \mathrm{s}$ are taken from the experiment [3]. Following Ref. [3] we consider dipole clusters consisting of $N=29,30,34$ particles which have different types of packing. In the ordered state the systems of $N=29,30$ particles are arranged in a triangular "closed-packed" structure having, respectively, the shell structure (3:9:17) and (3:9:18) and ground-state energy $E=2.2447 E_{0}$ and $E=2.2798 E_{0}$. The cluster with $N=34$ particles (4:11:19) $E=2.4198 E_{0}$ has a non-close-packed structure.

We find first the ground-state configuration using the Monte Carlo (MC) technique. Our results for the minimal energy configuration coincides exactly with the energy found in [5] for Coulomb clusters. In the experiment [3], the system was first equilibrated and after that the particle trajectories were recorded during $30 \mathrm{~min}$. In the simulation, we equilibrated the system for about $\left(5 \times 10^{5}-10^{6}\right)$ MC steps after which we started with the statistical averaging over time of the different observables. To obtain reliable results with small statistical error, we follow the particle trajectories typically during $10^{7}$ time steps.

We calculated the time dependence of the mean angular displacement of the particles in a specific shell $\theta(t)=$ $\sum_{i=1}^{N_{s}}\left[\theta_{i}(t)-\theta_{i}(0)\right] / N_{s}$, where $\theta_{i}(t)$ is the angular position of the $i$ th particle and $N_{s}$ is the number of particles in the shell. In Fig. 1 the angular displacements of the particles in the first shell (the most inner) and the second shell are given as functions of time for the cluster of $N=29$ particles. The angular motion in the third shell is very small because its motion is hindered by the hard wall. Notice that both the first (thick curve) and the second (thin curve) shells take part in the angular motion, but the former rotation is more prominent. The intershell motion has no preferential direction and with time it can be either a clockwise or a counterclockwise rotation. With decreasing coupling parameter $\Gamma$ intershell rotation becomes more pronounced.

In order to characterize the order of the system, we calculate the angular diffusion of the particles over a 30 min $\times 1000$ time interval. The angular diffusion coefficient can be written as

$$
D_{\theta}=\left[\left\langle\Delta \theta(t)^{2}\right\rangle-\langle\Delta \theta(t)\rangle^{2}\right] / t,
$$

where \langle\rangle refers to a time averaging, and the mean relative angular displacement rotation of the first shell $\left[\theta^{1}(t)\right]$
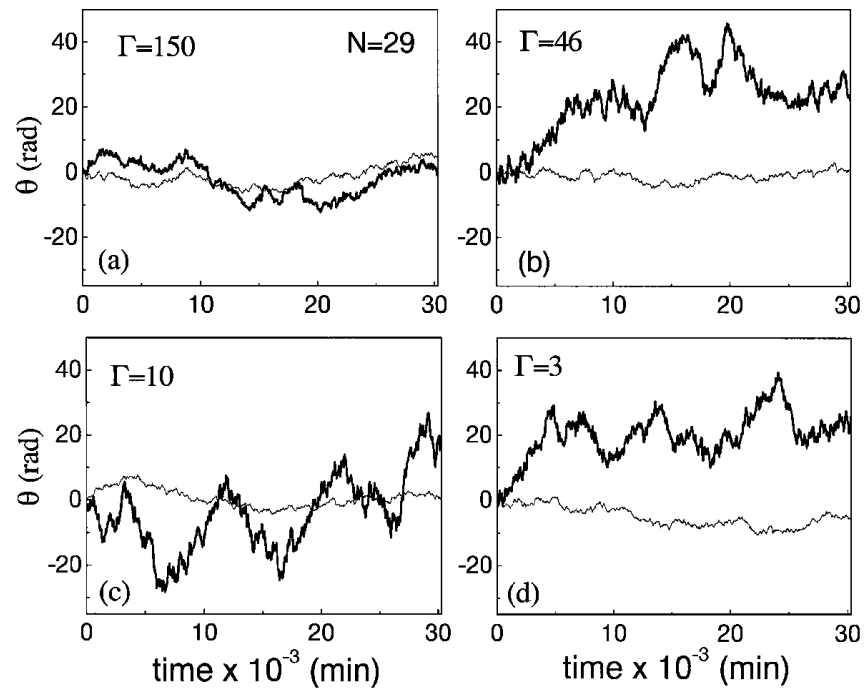

FIG. 1. The angular displacement of the particles on the first (thick curves) and second (thin curves) shell for a cluster with $N=29$ particles for different values of the coupling parameter: (a) $\Gamma=150$, (b) $\Gamma=46$, (c) $\Gamma=10$, and (d) $\Gamma=3$.

relative to the second $\left[\theta^{2}(t)\right]$ one is defined as $\Delta \theta(t)=$ $\theta^{1}(t)-\theta^{2}(t)$. The variance of the distribution function of the radial coordinates is

$$
\Delta R^{2}=\frac{1}{N} \sum_{i=1}^{N}\left[\left\langle r_{i}(t)^{2}\right\rangle-\left\langle r_{i}(t)\right\rangle^{2}\right] / a_{0}^{2},
$$

which is a measure of the radial order in the system.

In Fig. 2 the angular diffusion coefficient and the radial variance are shown for three different dipole clusters with hard wall confinement subjected to the same conditions as in the experiment [3]. For the cluster with $N=29$ particles, the angular diffusion [solid dots in Fig. 2(a)] monotonically increases with decreasing coupling parameter up to $\Gamma \sim 30$ which is a manifestation of angular melting. In the interval $\Gamma=10-30$ the intershell diffusion remains practically constant, and with further decreasing $\Gamma$ it is reduced to about a $20 \%$ smaller value. In the latter region the radial fluctuations start to rise [open dots in Fig. 2(a)], but the cluster retains its shell structure. In the range $3<\Gamma<8$ the cluster oscillates between the ground state (3:9:17) and the metastable state (4:8:17) which leads to a reduction of the angular fluctuations. Further decreasing the coupling beyond $\Gamma \approx 5$ both $D_{\theta}$ and $\Delta R^{2}$ rises quickly, indicating the onset of melting. A similar qualitative behavior was observed for the dipole cluster with $N=30$ particles [see Fig. 2(b)]. In the non-close-packed cluster with $N=34$, intershell rotation occurs over all $\Gamma$ 's considered in the experiment [see Fig. 2(c)] and no clear regaining of angular order is found in the region $3<\Gamma<8$.

In order to obtain further insight into this reentrant behavior, we investigated the conditions under which this novel effect can be observed. Therefore, we varied the following parameters: (i) the viscosity of the medium the 




where $\nu=k_{B} T / m D_{0}$ is the friction, and $\vec{F}_{i}$ is the force from the interparticle interaction. As an example, we consider $N=30$ dipole particles moving in a medium with a viscosity which is $10^{4}$ times smaller than the one of water. Such a low viscosity corresponds to the situation of colloidal particles moving in a gas with pressure $1 \mathrm{~Pa}$. In Fig. 2(b) the angular diffusion coefficient (squares) is plotted as function of $\Gamma$. Note that now $D_{\theta}$ is about a factor $10^{4}$ larger as compared to the previous case [see Fig. 2(b)]. It is clear that changing viscosity does not destroy the reentrantlike behavior but changes only the time scale for relaxation to equilibrium.

Next we investigated whether the type of interparticle interaction influences the occurrence of reentrant behavior. We consider a cluster with long-range Coulomb interaction $[N=37$ (3:9:25) having the same internal structure as the $N=30$ dipole cluster] with hard wall confinement using Brownian dynamics (1). In Fig. 3(a) the angular and radial diffusion coefficients are shown as a function of $\Gamma$. Notice that the Coulomb cluster shows completely different melting behavior and $D_{\theta}$ increases monotonically with decreasing $\Gamma$. Melting takes place at $\Gamma \sim 40$. Next we consider short-range interparticle interaction, and as an example we took the screened Coulomb cluster $\left(\kappa=2 / a_{0}\right)$ with $N=30$ (3:9:18) particles. In Fig. 3(b) the angular diffusion and radial variance are shown as a function of the coupling. $D_{\theta}$ exhibits a clear reentrant behavior which correlates with an increase of the radial diffusion. Thus we may conclude that only clusters with short-range interparticle interaction in a hard wall vessel show reentrant behavior.

Last we study the effect of the shape of the confinement potential and consider the melting behavior of a cluster with short-range interparticle interaction in a parabolic well. We choose the $N=25$ dipole cluster (3:9:13). Figure 4 shows the angular diffusion and the radius of the cluster as a function of $\Gamma$. $D_{\theta}$ clearly does not exhibit a local minimum, it rises uniformly with decreasing $\Gamma$, and melting occurs for $\Gamma \approx 5$, as in the case of hard wall confinement. The radius of the cluster $R$ and the mean interparticle distance changes proportionally to $\Gamma^{1 / 2}$.

In conclusion, we studied the melting transition of 2D

FIG. 2. The angular diffusion coefficient $D_{\theta}$ (solid circles) and the variance of the distribution function of the radial coordinates $\left(\Delta R^{2}\right)$ (open circles) coefficients as a function of $\Gamma$ for clusters with (a) $N=29$, (b) $N=30$, and (c) $N=34$ particles. The squares in (b) are $D_{\theta}$ for the case of small viscosity.

particles are moving in; (ii) the range of the interparticle interaction; and (iii) the form of the confinement potential.

To study the melting behavior of the cluster under the condition of low viscosity with hard wall confinement, we performed Langevin molecular dynamics simulations. The Langevin equations for the system of $N$ interacting particles are

$$
\frac{d^{2} \vec{r}_{i}}{d t^{2}}=-\nu \frac{d \vec{r}_{i}}{d t}+\frac{\vec{F}_{i}}{m}+\frac{\vec{F}_{L}}{m}
$$

clusters with dipole and screened Coulomb-type interaction confined by a hard wall or a parabolic external potential using Brownian dynamics simulations. Langevin molecular dynamics simulations were carried out in the case of small viscosity. We found that only clusters with short-range interparticle interaction and confined by a hard wall well exhibit angular freezing before melting, irrespective of the value of viscosity. In the other cases, either of Coulomb clusters or with parabolic confinement, the system shows the usual [5] two step melting behavior without any reentrance.

We showed that reentrant behavior is a consequence of the interplay between angular order and radial oscillations 

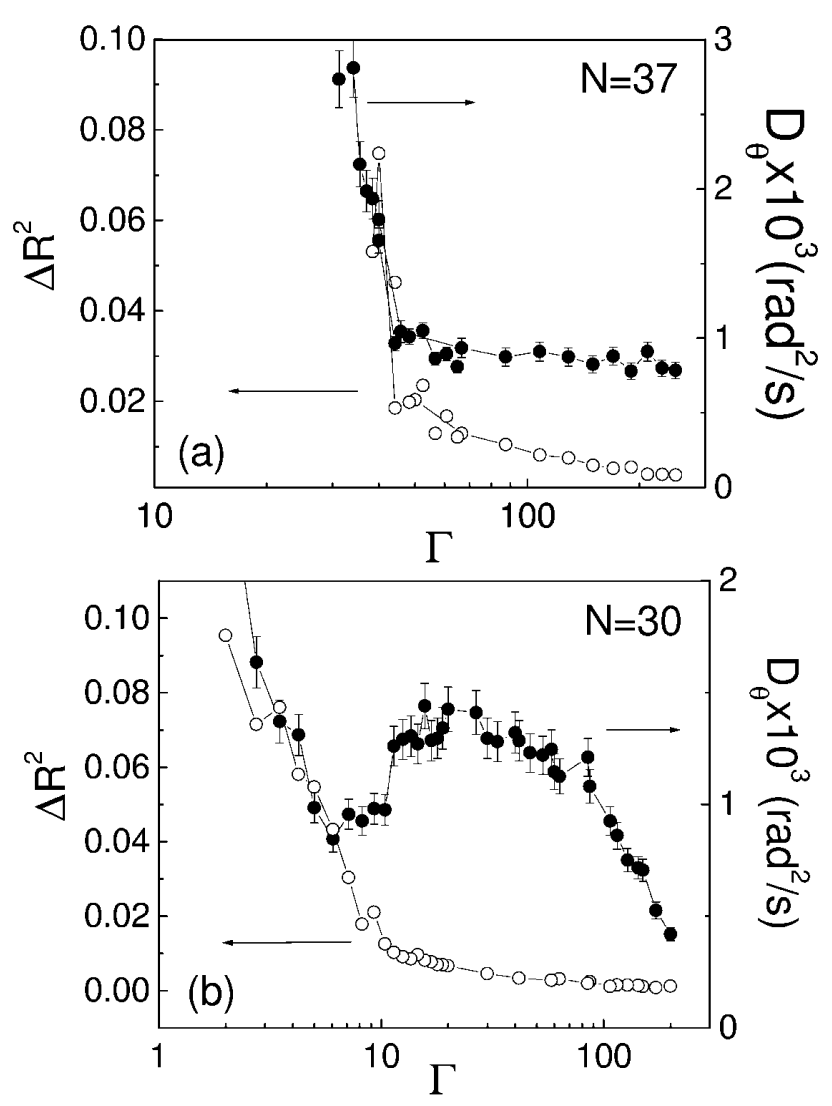

FIG. 3. The angular diffusion coefficient (solid circles) and the radial variance (open circles) as a function of $\Gamma$ for (a) the Coulomb cluster with $N=37$ particles, and (b) the screened Coulomb cluster with $N=30$ particles confined by a hard wall potential.

where an increase of the radial fluctuations is able to induce angular order in clusters with magic number. With decreasing $\Gamma$, first angular motion sets in, because it is governed by the lowest energy barriers. Further decreasing $\Gamma$ leads to an increase of the radial motion/fluctuations which hinder the angular motion. The latter prevents angular motion in case of hard wall confinement. But for parabolic confinement the average interparticle distance (see Fig. 4) decreases which results in a change of the energy barriers for intershell and intrashell motion. This contrasts with the hard wall confinement case where the interparticle distances are unaltered and the energy barrier for intershell jumps decreases leading to an increase of the radial fluctuations and of intershell jumps. Thus anharmonic effects are essential for the occurrence of this reentrant behavior which is enhanced in systems with hard wall confinement and for short-range interparticle interaction.

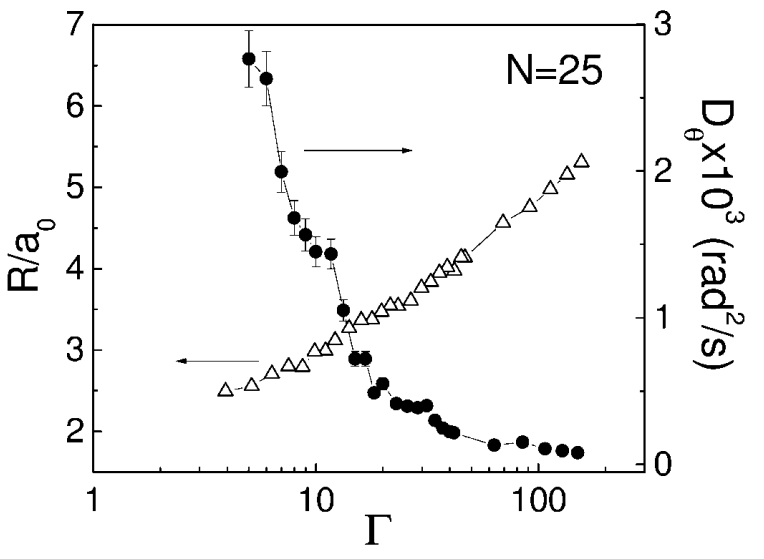

FIG. 4. The angular diffusion coefficient (solid circles) and radius of the cluster (open triangles) as a function of $\Gamma$ for a $N=25$ dipole cluster in a parabolic well.

One of us (F. M.P.) thanks C. Bechinger for fruitful discussions. This work is supported by the Flemish Science Foundation (FWO-Vl), GOA-UA, IUAP-IV, and INTAS-RFBR 97-775 (the Russian Foundation of Basic Researchers).

*Permanent address: Institute of Theoretical and Applied Mechanics, Russian Academy of Sciences, Novosibirsk 630090, Russia.

†Electronic mail: peeters@uia.ua.ac.be

[1] W.-T. Juan et al., Phys. Rev. E 58, R6947 (1998).

[2] D. J. Wineland et al., Phys. Rev. Lett. 59, 2935 (1987).

[3] R. Bubeck, C. Bechinger, S. Neser, and P. Leiderer, Phys. Rev. Lett. 82, 3364 (1999).

[4] Yu. E. Lozovik and V. A. Mandelshtam, Phys. Lett. A 145, 269 (1990).

[5] V. M. Bedanov and F. M. Peeters, Phys. Rev. B 49, 2667 (1994).

[6] V. A. Schweigert and F. M. Peeters, Phys. Rev. B 51, 7700 (1995).

[7] I. V. Schweigert, V. A. Schweigert, and F. M. Peeters, Phys. Rev. B 54, 10827 (1996).

[8] V. A. Schweigert and F. M. Peeters, J. Phys. Condens. Matter 10, 2417 (1998).

[9] L. Cândido, J.-P. Rino, N. Studart, and F. M. Peeters, J. Phys. Condens. Matter 10, 11627 (1998).

[10] J. Chakrabarti et al., Phys. Rev. Lett. 75, 2232 (1995).

[11] Q.-H. Wei et al., Phys. Rev. Lett. 81, 2606 (1998).

[12] D. L. Ermak and J. A. McCammon, J. Chem. Phys. 69, 1352 (1978). 